\title{
ORIGINAL
}

\section{SÍNDROME DE BURNOUT EN EL PERSONAL DE ENFERMERÍA DE UN HOSPITAL DE MADRID}

\section{Romana Albaladejo, Rosa Villanueva, Paloma Ortega, P Astasio, ME Calle y V Domínguez}

Departamento de Medicina Preventiva y Salud Pública e Historia de la Ciencia. Facultad de Medicina de la Universidad Complutense de Madrid. Madrid 28040.

\section{RESUMEN}

Fundamento: El término Burnout se refiere a una situación que es cada vez más frecuente entre los profesionales que prestan sus servicios a través de una relación directa y mantenida con las personas, entre los que se encuentran todos los profesionales sanitarios. E objetivo de nuestro estudio ha sido determinar la prevalencia del síndrome de Burnout y de sus tres componentes (cansancio emocional, despersonalización y falta de realización personal) entre los profesionales del área de enfermería del Hospital Clínico Universitario San Carlos de Madrid y su relación con determinados factores sociodemográficos, laborales e institucionales.

Métodos: Se realizó un estudio tranversal entre los profesionales adscritos al área de enfermería del mencionado hospital. Las variación propia. En cuanto al síndrome de Burnout se midió mediante el Maslach Burnout Inventory, en su versión validada en español.

Resultados: El personal de enfermería está más despersonalizado $(\mathrm{p}=0,004)$ y menos realizado $(\mathrm{p}=0,036)$ que el grupo de auxiliares/técnicos. Cuando se analizaron los resultados de las 4 escalas po servicios comprobamos que el agotamiento es superior en los trabajadores de oncología y urgencias $(\mathrm{p}=0,001)$, la despersonalización en urgencias $(\mathrm{p}=0,007)$, y el Burnout es de nuevo más alto en las áreas de oncología y urgencias $(\mathrm{p}=0,000)$. Los profesionales que respondí an que su labor asistencial era poco reconocida obtenían las peores puntuaciones en el Burnout y sus tres dimensiones $(p=0,000)$. A menor grado de satisfacción laboral más altas son las puntuaciones en las 4 escalas $(\mathrm{p}=0,000)$.

Conclusiones: Podemos concluir que en nuestro estudio el perfil del sujeto afecto de Burnout se corresponde con el de un profesiota un escaso grado de reconocimiento a su labor asistencial y un alto nivel de insatisfacción con la gestión de su centro de trabajo.

Palabras clave: Agotamiento profesional. Enfermeras. Demografía. Satisfacción en el trabajo. Hospitales.

Correspondencia:

Departamento de Medicina Preventiva y Salud Pública.

Facultad de Medicina.

Universidad Complutense de MadrId.

Avenida Complutense, $\mathrm{s} / \mathrm{n}$

Madrid 28040.

Correo electrónico: mrvillan@med.ucm.es

\section{ABSTRACT}

\section{Burnout Syndrome among Nursing Staff at a Hospital in Madrid}

Background: The term «burnout» is related to a situation arising increasingly more often among the professionals performing their duties by way of a long-term, direct, people-to-people relationship, which includes all healthcare professionals. This study is aimed at determining the prevalence of the Burnout syndrome and of the three components involved therein (emotional exhaustion, of the three components involved therein (emotional exhaustion,
impersonalization and lack of personal fulfillment) among the nursing staff at the «Hospital Clínico Universitario San Carlos» in Madrid and the relationship thereof to certain socio-demographic job-related and institutional factors.

Methods: A cross-sectional study was conducted among the professionals assigned to the nursing staff at the above-mentioned hospital. The variables involved were gathered by means of a questionnaire prepared by those conducting this study. The Burnout syndrome was measured by means of the Maslach Burnout Inventory, in the validated Spanish version thereof

Results: The nursing staff is more impersonalized $(\mathrm{p}=0.004)$ and less fulfilled $(\mathrm{p}=0.036)$ than the nursing assistant/technician group. When the results of the four scales by units were analyzed, burnou was found to be greater among the nursing staff assigned to oncology and emergency care units $(\mathrm{p}=0.001)$, the impersonalization in the emergency rooms $(\mathrm{p}=0,007)$, and Burnout is once again greater in emergency rooms $(\mathrm{p}=0,007)$, and Burnout is once again greater
the oncology and emergency units $(\mathrm{p}=0.000)$. Those professionals who answered that there was little recognition of their nursing care scored worst regarding Burnout and the three aspects thereof $(p$ $=0.000)$. The lower the degree of on-the-job satisfaction, the higher the scores on the four scales $(\mathrm{p}=0.000)$.

Conclusions: The conclusion which may be drawn from this study is that the profile of a person affected by Burnout is that of a professional with on-the-job experience who nevertheless considers very little recognition to be given to their caregiving and a high degree of dissatisfaction with the way in which their workplaces are managed.

Key words: Burnout, professional. Nurses. Demography. Job satisfaction. 


\section{INTRODUCCIÓN}

El estrés forma parte de nuestra vida cotidiana hasta tal punto que puede considerarse como «el malestar de nuestra civilización», afectando tanto a la salud y al bienestar personal como a la satisfacción laboral y colectiva. En el contexto de la salud laboral surge el desarrollo de un nuevo proceso: el síndrome de Burnout. Diversos autores han documentado que este síndrome afecta más a aquellas profesiones que requieren un contacto directo con las personas y con una «filosofía humanística» del trabajo, es decir aquéllas que necesitan altas dosis de entrega e implicación. Así, son muchos los profesionales potencialmente afectados por el síndrome (enfermeros, médicos, trabajadores sociales, profesores, policías), y además está muy extendido dentro de cada uno de ellos ${ }^{1-3}$.

Maslach y Jackson ${ }^{4}$ definieron el Burnout como «una pérdida gradual de preocupación y de todo sentimiento emocional hacia las personas con las que trabajan y que conlleva a un aislamiento o deshumanización». Por otro lado, sentaron las bases para la realización de estudios en diferentes colectividades y sociedades, ya que idearon un instrumento de medida, denominado Maslach Burnout Inventory $(\mathrm{MBI})^{4,5}$.

Además, estas autoras identificaron los tres aspectos o dimensiones del Burnout: el cansancio emocional, la despersonalización y la falta de realización personal. El cansancio emocional se ha definido como desgaste, pérdida de energía, agotamiento y fatiga, que puede manifestarse física, psicológicamente o como una combinación ${ }^{4}$.

El segundo aspecto relatado es la tendencia a la despersonalización, por la que aparece un cambio negativo en las actitudes hacia otras personas, especialmente hacia los beneficiarios del propio trabajo, a los cuales se puede considerar como meros números («el 714»). En este sentido, se ha argumentado que la despersonalización en niveles moderados sería una respuesta adaptativa para los profesionales, pero esta misma respuesta, en grado excesivo, demostraría sentimientos patológicos hacia los otros, de insensibilidad y cinismo ${ }^{6}$. El tercer componente del Burnout es el sentimiento de falta de realización personal, por el que aparecen una serie de respuestas negativas hacia uno mismo y a su trabajo, típicas de depresión, moral baja, incremento de la irritabilidad, evitación de las relaciones profesionales, baja productividad, incapacidad para soportar la tensión, pérdida de la motivación hacia el trabajo y baja autoestima ${ }^{4}$.

Para otros autores ${ }^{7}$ el Burnout se refiere a un proceso en el que los profesionales están desengañados de su trabajo, en respuesta a experiencias de estrés y tensión en el mismo. Los problemas que pueden ser solventados no llevan al Burnout, de hecho el estrés controlado puede tener efectos positivos, estimulando al individuo a superarse, lo que culminaría en una experiencia gratificante y en competencia profesional. Precisamente, el Burnout comenzaría cuando el individuo se siente más indefenso para cambiar la situación ${ }^{8}$.

De todo lo anterior se deriva que para que aparezca el Burnout es necesaria la interacción entre las respuestas individuales al estrés junto a la presión laboral en el ambiente de trabajo9 ${ }^{9}$. En este marco el personal sanitario, y muy especialmente la enfermería, sería particularmente vulnerable al Burnout. Así, según estudios epidemiológicos recientes realizados en el personal de enfermería, hasta un $25 \%$ del mismo estaría afectado por el síndrome ${ }^{10}$. En el clásico estudio de House en las enfermeras ${ }^{11}$, se identificaban como características negativas del ambiente laboral la carga de trabajo, la ambigüedad del rol a desempeñar y la baja autoestima o valoración profesional.

Por un lado, el trabajo que se desarrolla en los hospitales requiere que todos los profesionales tengan la suficiente experiencia clí- 
nica y madurez que les permita enfrentarse a la toma de decisiones difíciles, de las que se derivan frecuentemente implicaciones éticas y morales. En este sentido, la enfermería se ha identificado como un grupo especial de riesgo porque en ella concurren factores personales, profesionales e institucionales, que son causas potenciales del síndrome que estudiamos ${ }^{4}$.

Maslach ${ }^{4}$ agrupa las causas responsables del síndrome en tres categorías de factores (personales, profesionales e institucionales) que se dan cita en el personal de enfermería, favoreciendo la aparición del cuadro en este grupo.

En cuanto a los aspectos epidemiológicos del síndrome de Burnout descritos en la literatura no parece existir un acuerdo unánime entre los distintos autores, si bien existe un determinado nivel de coincidencia para algunas variables. La edad no parece influir y según la variable sexo, serían las mujeres sanitarias las más afectadas, quizás por el mantenimiento de la doble carga de trabajo familiar y profesional ${ }^{12}$.

Otros factores, sobre los que existe más controversia, serían los cambios de turnos y los horarios laborales, el salario, la categoría profesional y la sobrecarga laboral asistencial.

Además, siguiendo la bibliografía existente, se detectan una serie de áreas de trabajo con mayor incidencia del síndrome, como es el caso de los servicios de urgencia y Unidad de Cuidados Intensivos (UCI) ${ }^{5,13-15}$. Las características del trabajo que se desarrolla en estas unidades requieren notable experiencia clínica y madurez profesional que permita hacer frente a la frecuente toma de decisiones difíciles con implicaciones éticas y morales.

Otro grupo de riesgo especial es el de los trabajadores del área de oncología, donde la enfermería se enfrenta diariamente al sufri- miento humano y en muchos casos a los pacientes terminales y a la muerte ${ }^{16}$.

Al considerar los factores institucionales diferentes autores ${ }^{11,17}$ destacan positivamente el soporte social como el principal medio para contrarrestar los efectos del Burnout. Por el contrario, se han identificado numerosos factores estresantes crónicos del entorno laboral implicados en su producción, como los pacientes difíciles, bien por padecer patologías graves, bien por su propio inconformismo; el poco tiempo por paciente y la mala calidad de las relaciones de equipo ${ }^{18-22}$.

Dado que no se dispone de suficientes estudios que aporten datos de la prevalencia de Burnout en Madrid y que el personal que trabaja en los grandes hospitales está, según los estudios realizados previamente, «a priori» más expuesto a padecer este proceso, el objetivo de este trabajo ha sido determinar la prevalencia del síndrome de Burnout en los profesionales adscritos al área de enfermería de un hospital. Asimismo, nos hemos propuesto conocer la distribución de este síndrome en función de la categoría profesional y de las áreas de trabajo a las que pertenecen.

\section{SUJETOS Y MÉTODOS}

Se realizó un estudio transversal, eligiendo a los participantes mediante muestreo aleatorio estratificado por los distintos servicios hospitalarios, entre todo el personal adscrito al Área de Enfermería del Hospital Clínico Universitario de San Carlos. La muestra de estudio la constituyeron 622 profesionales clasificados en dos categorías: enfermeros/as y auxiliares/técnicos. Finalmente se clasificaron en cuatro grupos en función del riesgo específico conocido previamente para la aparición del síndrome (oncología-hematología oncológica, urgencias, UCI, grupo general $)^{16}$.

Para la recogida de la información se han aplicado dos tipos de instrumentos. En pri- 
mer lugar un cuestionario de elaboración propia, por medio del cual se recogieron las variables sociodemográficas (edad, sexo, categoría profesional, servicio, estado civil, número de hijos, antigüedad en la profesión y en el puesto de trabajo, situación y turno laboral, número de enfermos a su cargo). Dicho cuestionario incluía además cinco preguntas con el fin de valorar sus opiniones personales sobre las causas y las consecuencias de su posible malestar en el trabajo ${ }^{23}$. Estas cinco cuestiones versaban sobre:

- la opinión del propio trabajador sobre el grado en que ve reconocido su esfuerzo y labor asistencial por la dirección del centro (Pregunta 1).

- la opinión del propio trabajador sobre el grado en que es reconocido su trabajo por los pacientes (Pregunta 2).

- el grado de satisfacción del trabajador con su situación laboral actual (Pregunta 3 ).

- su propia valoración de la política de personal y de la gestión de recursos humanos (Pregunta 4).

- el grado de identificación del trabajador con la empresa (Pregunta 5).

Estas preguntas se valoraron según la escala de Likert con una puntuación de 1 a 5 , de forma que las puntuaciones inferiores a 3 traducían sentimientos positivos, 3 indicaba un nivel intermedio y valores superiores a 3 expresaban respuestas negativas respecto a las cuestiones planteadas.

La segunda herramienta empleada en la recogida de la información fue el Maslach Burnout Inventory (MBI), en su versión validada en español ${ }^{6,23}$. Este cuestionario mide las tres dimensiones del Síndrome de Burnout: el cansancio emocional (CE), la despersonalización (DP) y la realización personal (RP).
La primera de estas dimensiones, el cansancio emocional, se recoge mediante ocho preguntas con cuatro posibles respuestas, valoradas según la escala de Likert. La despersonalización se valora mediante cinco preguntas cuantificadas igual que la anterior, y finalmente la dimensión de realización personal se mide por medio de ocho items con las mismas condiciones anteriores. A la hora de interpretar los datos en el caso de las dos primeras escalas la mayor puntuación indica peor resultado mientras que en la tercera dimensión (realización personal) la interpretación es a la inversa.

Finalmente, utilizamos una cuarta escala a la que hemos llamado Burnout, que se obtenía sumando las puntuaciones de las tres dimensiones, cuyo resultado es directamente proporcional en el caso del cansancio emocional y la despersonalización e inversamente proporcional en la escala de realización personal. De esta manera se considera Burnout cuando se puntúa alto en cansancio emocional y despersonalización y bajo en realización personal ${ }^{23,25}$.

También, teniendo en cuenta los puntos de corte establecidos previamente en la literatu$\mathrm{ra}^{22}$, categorizamos las cuatro escalas en niveles bajos, medios o altos, de cansancio emocional $(<17 ; 18-26$; $>27)$, despersonalización $(<8 ; 9-12 ;>13)$, realización personal $(<16$; $17-22 ;>23)$ y Burnout $(<38 ; 39-51 ;>52)$.

El análisis de los datos se efectuó utilizando el paquete estadístico SPSSPC V10.0. Se realizó un estudio estadístico descriptivo y analítico, siendo el nivel de significación elegido de $\mathrm{p}<0,05$.

\section{RESULTADOS}

\section{Datos sociodemográficos y laborales y de opinión sobre el malestar laboral}

La población real estaba constituida por 622 profesionales, de los cuales el 58,7\% 
trabajaban como personal de enfermería y el $41,3 \%$ como auxiliares/técnicos. El sexo era mayoritariamente femenino (un $89,1 \%$ respecto a un $10,9 \%$ de varones), y la edad media de la población fue de 38,96 $\pm 9,65$ años.

En cuanto al estado civil el 54,8\% estaban casados o tenían pareja fija, el 36,5\% eran solteros, un $7,1 \%$ separados o divorciados y un $1,1 \%$ viudos. El $50,5 \%$ tenían hijos siendo la media por hogar de 0,93 .

Respecto a las variables laborales y de opinión hallamos que el tiempo medio de

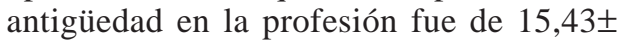
8,96 y en el puesto de trabajo de 7,70 $\pm 7,53$. El $67,2 \%$ de los participantes en el estudio desempeñaban su puesto de trabajo en calidad de fijos, el 27,3\% con contrato indefinido/interino y un $4,8 \%$ realizaba suplencias. Al considerar su horario, de trabajo hallamos que el $35 \%$ trabajaba en el turno de mañana, el $18,2 \%$ por la tarde, el $10,1 \%$ por la noche, un $34,6 \%$ rotaba y el $1,2 \%$ desempeñaban turnos de 12 ó 24 horas.

Finalmente, en cuanto a la carga asistencial el número medio de enfermos por profesional fue de 14,31 .
En la tabla 1 se recogen los resultados obtenidos sobre satisfacción y calidad en la gestión de personal según los servicios. Fueron semejantes en todos los grupos salvo en «identificación con la organización», que resultó peor valorada, de forma estadísticamente significativa, en el sentido de que fue peor (mayor puntuación) entre los profesionales de oncología.

\section{Resultados obtenidos en el Burnout y sus subescalas}

Los valores obtenidos en las distintas subescalas del MBI fueron de 19,61 $\pm 5,54$ en la de agotamiento; de 8,37 $\pm 2,66$ en la de despersonalización; de 20,80 $\pm 4,25$ en la de realización personal y de 46,97士9,68 en la de Burnout.

La frecuencia de personal sanitario afectado por niveles bajos, medios y altos, de acuerdo con las cifras mencionadas en material y métodos, de cansancio emocional fue de $40 \%, 48,2 \%$ y $11,7 \%$; de $57,9 \%, 32,8 \%$ y $9,2 \%$ para la despersonalización; de $35 \%$, $48,1 \%$ y $16,9 \%$ para la falta de realización personal, y finalmente, de $20,6 \%, 48,9 \%$ y $30,5 \%$ para el Burnout.

Tabla 1

Resultados de las 5 preguntas de opinión sobre la satisfacción y calidad de gestión de personal según servicios hospitalarios

\begin{tabular}{|c|c|c|c|c|c|}
\hline & Oncología & UCI & Urgencias & General & $\mathbf{p}$ \\
\hline $\begin{array}{l}\text { Reconocimiento del } \\
\text { trabajo por la dirección }\end{array}$ & $4,29 \pm 0,90$ & $4,10 \pm 0,84$ & $4,08 \pm 0,98$ & $3,95 \pm 1,08$ & 0,117 \\
\hline $\begin{array}{l}\text { Reconocimiento del } \\
\text { trabajo por los pacientes }\end{array}$ & $2,50 \pm 0,74$ & $2,56 \pm 0,75$ & $2,98 \pm 0,85$ & $2,63 \pm 0,89$ & 0,060 \\
\hline $\begin{array}{l}\text { Satisfacción personal con } \\
\text { su trabajo }\end{array}$ & $3,42 \pm 0,96$ & $2,95 \pm 0,97$ & $3,47 \pm 1,04$ & $3,21 \pm 0,98$ & 0,070 \\
\hline Políticas de personal & $4,42 \pm 0,74$ & $4,05 \pm 0,88$ & $4,28 \pm 0,74$ & $4,22 \pm 0,77$ & 0,058 \\
\hline $\begin{array}{l}\text { Identificación con la } \\
\text { organización }\end{array}$ & $3,42 \pm 1,01$ & $2,92 \pm 1,07$ & $3,19 \pm 0,91$ & $3,05 \pm 0,96$ & 0,028 \\
\hline
\end{tabular}


Tabla 2

Resultados en las escalas de cansancio emocional, despersonalización, realización personal y Burnout, según las opiniones del trabajador acerca del reconocimiento de su labor por la organización (Pregunta 1) y por los pacientes (Pregunta 2)

\begin{tabular}{|c|c|c|c|c|}
\hline & $\begin{array}{l}\text { Cansancio } \\
\text { emocional }\end{array}$ & Depersonalización & Realización personal & Burnout \\
\hline \multicolumn{5}{|c|}{$\begin{array}{l}\text { Satisfacción con el trabajo } \\
\text { actual }\end{array}$} \\
\hline Siempre & $15,47 \pm 3,68$ & $7,07 \pm 1,83$ & $23,93 \pm 4,33$ & $38,53 \pm 8,75$ \\
\hline Casi siempre & $15,88 \pm 3,84$ & $7,24 \pm 1,94$ & $22,06 \pm 4,42$ & $40,85 \pm 7,48$ \\
\hline Algunas veces & $17,21 \pm 4,36$ & $7,59 \pm 2,34$ & $22,18 \pm 4,01$ & $42,30 \pm 7,67$ \\
\hline Ocasionalmente & $19,14 \pm 5,04$ & $8,39 \pm 2,66$ & $20,36 \pm 3,87$ & $47,06 \pm 8,76$ \\
\hline Nunca & $22,00 \pm 5,67$ & $9,03 \pm 2,76$ & $19,96 \pm 4,29$ & $50,81 \pm 9,75$ \\
\hline $\mathrm{p}$ & 0,000 & 0,000 & 0,000 & 0,000 \\
\hline \multicolumn{5}{|c|}{$\begin{array}{l}\text { Reconocimiento del } \\
\text { trabajo por los pacientes }\end{array}$} \\
\hline Siempre & $16,91 \pm 5,07$ & $6,98 \pm 1,83$ & $23,23 \pm 5,04$ & $40,79 \pm 9,52$ \\
\hline Casi siempre & $18,61 \pm 5,31$ & $7,94 \pm 2,44$ & $21,35 \pm 3,99$ & $44,97 \pm 8,97$ \\
\hline Algunas veces & $19,94 \pm 5,44$ & $8,73 \pm 1,68$ & $20,14 \pm 4,04$ & $48,25 \pm 9,30$ \\
\hline Ocasionalmente & $22,77 \pm 5,35$ & $9,20 \pm 1,93$ & $19,88 \pm 4,53$ & $52,12 \pm 9,06$ \\
\hline Nunca & $22,79 \pm 4,74$ & $9,40 \pm 1,45$ & $19,80 \pm 4,26$ & $52,10 \pm 8,08$ \\
\hline $\mathrm{p}$ & 0,000 & 0,000 & 0,000 & 0,000 \\
\hline
\end{tabular}

Por otro lado, cuando se analizaron los datos obtenidos en estas escalas según sexo, estado civil, turno laboral y tipo de contrato, encontramos diferencias estadísticamente significativas según el sexo, en cansancio emocional, despersonalización y Burnout, siendo las puntuaciones superiores en las mujeres $(\mathrm{p}=0,014, \mathrm{p}=0,029, \mathrm{p}=0,008$ respectivamente). En cuanto al estado civil los casados tuvieron las puntuaciones más altas en cansancio emocional, siendo las diferencias entre grupos estadísticamente significativas $(\mathrm{p}=0,004)$.

También se hallaron puntuaciones más altas respecto al cansancio emocional, la des- personalización y el Burnout en los profesionales que trabajaban en turno de tarde o rotatorios $(\mathrm{p}=0,013, \mathrm{p}=0,001, \mathrm{p}=0,004$, respectivamente). Por el contrario, los profesionales que realizaban suplencias estaban menos despersonalizados, más realizados y menos «quemados» $(p=0,011, p=0,010, p=0,009)$.

Al estudiar el Burnout y sus componentes según la categoría profesional (enfermería versus auxiliares/técnicos) se vió que el primero estaba más despersonalizado $(p=0,004)$ y menos realizado $(p=0,036)$ que el grupo de los auxiliares/técnicos, no encontrándose otras diferencias estadísticamente significativas. 
Tabla 3

\begin{tabular}{|c|c|c|c|c|}
\hline & $\begin{array}{l}\text { Cansancio } \\
\text { Emocional }\end{array}$ & Depersonalización & $\begin{array}{l}\text { Realización } \\
\text { personal }\end{array}$ & Burnout \\
\hline \multicolumn{5}{|c|}{$\begin{array}{l}\text { Satisfacción con el trabajo } \\
\text { actual }\end{array}$} \\
\hline Muy satisfecho & $13,88 \pm 3,95$ & $7,03 \pm 1,38$ & $23,79 \pm 3,37$ & $37,56 \pm 7,64$ \\
\hline Bastante satisfecho & $15,91 \pm 4,02$ & $7,61 \pm 1,26$ & $22,25 \pm 3,87$ & $40,70 \pm 7,15$ \\
\hline Satisfecho & $18,00 \pm 4,13$ & $7,94 \pm 1,50$ & $21,36 \pm 3,01$ & $44,36 \pm 7,80$ \\
\hline No muy satisfecho & $22,69 \pm 4,52$ & $9,21 \pm 1,68$ & $19,41 \pm 4,12$ & $52,47 \pm 8,21$ \\
\hline Insatisfecho & $26,67 \pm 4,92$ & $9,77 \pm 2,04$ & $18,53 \pm 4,29$ & $57,40 \pm 8,46$ \\
\hline $\mathrm{p}$ & 0,000 & 0,000 & 0,000 & 0,000 \\
\hline \multicolumn{5}{|l|}{ Políticas de personal } \\
\hline Muy buena & $18,00 \pm 4,21$ & $8,67 \pm 1,21$ & $19,67 \pm 3,06$ & $30,33 \pm 3,51$ \\
\hline Bastante buena & $15,30 \pm 3,47$ & $6,80 \pm 1,14$ & $24,80 \pm 3,49$ & $37,60 \pm 5,56$ \\
\hline Buena & $16,23 \pm 3,95$ & $7,36 \pm 1,22$ & $22,57 \pm 3,83$ & $41,00 \pm 7,69$ \\
\hline Regular & $18,33 \pm 4,56$ & $8,08 \pm 1,56$ & $21,11 \pm 4,04$ & $45,08 \pm 8,53$ \\
\hline Pobre & $22,53 \pm 5,61$ & $9,17 \pm 1,72$ & $19,73 \pm 4,25$ & $51,77 \pm 9,31$ \\
\hline $\mathrm{p}$ & 0,000 & 0,000 & 0,000 & 0,000 \\
\hline \multicolumn{5}{|l|}{$\begin{array}{l}\text { Identificación con la } \\
\text { organización }\end{array}$} \\
\hline Muy identificado & $16,85 \pm 3,39$ & $7,03 \pm 1,08$ & $22,87 \pm 4,53$ & $41,00 \pm 8,64$ \\
\hline Bastante identificado & $16,76 \pm 3,69$ & $7,50 \pm 1,37$ & $22,83 \pm 4,18$ & $41,25 \pm 8,92$ \\
\hline Identificado & $18,57 \pm 4,70$ & $8,10 \pm 1,38$ & $20,89 \pm 2,55$ & $45,48 \pm 7,34$ \\
\hline Poco identificado & $22,67 \pm 5,36$ & $9,34 \pm 1,93$ & $19,56 \pm 2,12$ & $52,38 \pm 9,47$ \\
\hline No identificado & $25,22 \pm 5,11$ & $10,10 \pm 1,82$ & $17,61 \pm 3,10$ & $57,46 \pm 8,54$ \\
\hline $\mathrm{p}$ & 0,000 & 0,000 & 0,000 & 0,000 \\
\hline
\end{tabular}

Cuando se analizaron los resultados de las 4 escalas por servicios, comprobamos que el agotamiento era estadísticamente mayor en los trabajadores de oncología y urgencias 
$(\mathrm{p}=0,001)$, la despersonalización en el personal de urgencias $(\mathrm{p}=0,007)$, no había diferencias en cuanto a la realización personal, y el Burnout era, de nuevo, estadísticamente más alto en las áreas de oncología y urgencias $(\mathrm{p}=0,000)$.

Al analizar el Burnout y sus subescalas según opiniones expresadas en las preguntas 1 a 5 (tabla 2), se comprobó que aquellos profesionales que respondían que su labor asistencial era poco reconocida por la dirección del centro y los enfermos, obtenían las peores puntuaciones en las tres subescalas y en el Burnout.

Cuando se analizó la relación entre el grado de satisfacción en el trabajo actual (pregunta 3), la opinión sobre la política de personal (pregunta 4) y el grado de identificación con el centro de trabajo (pregunta 5) y las escalas del «quemado» (tabla 3 ), se verificó que cuando el grado de satisfacción, la opinión sobre la política de personal y el grado de identificación con el centro de trabajo eran malas se obtenían puntuaciones significativamente más altas, en cuanto al agotamiento, la despersonalización y el Burnout y más bajas en cuanto al grado de realización personal.

\section{DISCUSIÓN}

En primer lugar, vamos a comenzar con una serie de reflexiones sobre el instrumento de medida utilizado en este trabajo de investigación: el Maslach Burnout Inventory ${ }^{4}$. Así, hay que considerar que desde el desarrollo original de esta escala se han realizado diferentes modificaciones con el fin de facilitar su aplicación, pasándose de las 47 preguntas iniciales a las 22 actuales.

En este sentido, a lo largo del tiempo, se ha discutido la estructura trifactorial del cuestionario (el estudio del cansancio emocional, la despersonalización y la falta de realización personal), afirmando que una ampliación de la misma conduciría al mejor conocimiento del problema del «quemado». Por el contrario, otros autores ${ }^{6}$ sugieren que es más replicable e interpretable la información recogida a través de un pequeño número de factores, y que además ésta no mejoraría con la incorporación de otras escalas.

En esta última línea de pensamiento se encuentra la adaptación española del $\mathrm{MBI}^{24}$, que ha sido la utilizada en nuestro trabajo de investigación. No obstante, hemos querido comprobar su reproducibilidad y para ello hemos medido la consistencia interna de las escalas, mediante los coeficientes alfa de Cronbach estandarizados, obteniéndose unos valores de: 0,9089 para cansancio emocional; 0,7532 para despersonalización; 0,8133 para realización personal y 0,6870 para Burnout total. Debemos reseñar que estos valores se hallan dentro del rango considerado como válido previamente en la literatura $^{25}$

En segundo lugar y en relación con el tema que nos atañe, debemos resaltar que en la actualidad nos encontramos con una bibliografía llena de aportaciones referentes a la importancia del síndrome de Burnout en las profesiones sanitarias (médicos/as, enfermeros/as, auxiliares $)^{10,12,26-30}$. A pesar de ello, sigue existiendo gran disparidad de resultados en cuanto a las prevalencias del Burnout en general y de cada uno de sus tres componentes en particular. En nuestro caso, como hemos explicado anteriormente, encontramos prevalencias en rangos bajosmedios para cansancio emocional y despersonalización; y rangos medios-altos en falta de realización personal y Burnout.

En este sentido debemos recordar que tal como expresó Freudenberger ${ }^{31}$, la inclusión de los tres aspectos o dimensiones del Burnout antes mencionados enfatiza la complejidad del mismo. Sin embargo, tal planteamiento no significa que el síndrome no sea un concepto unitario, sólo muestra una variedad de reacciones psicológicas que los 
trabajadores pueden experimentar con este proceso y que podría explicar las diferencias encontradas en estas prevalencias ${ }^{4}$.

Ante estos datos, cabe preguntarse qué factores influyen con mayor frecuencia en la aparición del Burnout en las profesiones sanitarias $^{32}$. Así, en lo que se refiere a los factores personales nuestro estudio muestra un grupo bastante homogéneo: mayoritariamente femenino, casados o con pareja fija y con una media de hijos acorde a las estadísticas actuales en nuestro país. En cuanto a las variables laborales encontramos que la gran mayoría tienen un puesto de trabajo fijo y una media de antigüedad en la profesión de unos 15 años, perfil bastante semejante al encontrado por otros autores que han estudiado el problema ${ }^{29,33,34}$.

Respecto a la edad tenemos que resaltar que si bien no se ha descrito que intervenga en la aparición del síndrome, en relación directa con la misma estaría la antigüedad profesional. Diferentes autores han indica$\mathrm{do}^{32}$ que después de 10 años puede existir un período de sensibilización a partir del cual el profesional sería especialmente vulnerable. Así, tras este tiempo de ejercicio profesional se ha producido ya la transición de las expectativas idealistas hacia la práctica cotidiana, viéndose que tanto las recompensas personales como las profesionales y las económicas no son ni las prometidas ni las esperadas.

La edad no tuvo relación con ninguna de las variables estudiadas. Sin embargo, sí se obtuvieron diferencias estadísticamente significativas según sexo, estado civil, turno y tipo de contrato. En cuanto a esta última variable resulta curioso que se han obtenido mejores resultados en el Burnout y sus subescalas entre los profesionales que realizaban suplencias. Este dato se podría explicar por el hecho de que se trata de sujetos más jóvenes y con menor tiempo en el puesto de trabajo y, por tanto, tal como recogen otros autores, menos susceptibles al síndrome de Burnout ${ }^{5}$.
Por otro lado, entre los factores profesionales también es conocida la relación de la presencia del Burnout y los distintos servicios hospitalarios ${ }^{4,5,13-15}$. Se sabe que en todas las áreas clínicas y especialmente en servicios como oncología y unidades de cuidados intensivos y urgencias, tal como demuestran los estudios previos, se requieren unas aptitudes que no todos poseen: una dedicación y vocación muy definidas, estabilidad psicológica y emocional, y una salud física y capacidad de trabajo que permita hacer frente a la gran carga asistencial y emocional de estas unidades ${ }^{16}$. En este sentido los datos de nuestro estudio concuerdan con los de la literatura mencionados previamente, mostrando que el agotamiento, la despersonalización y el Burnout eran significativamente más altos entre el personal de los servicios de urgencias y oncología.

Otro punto en el que coincidimos con los expertos en el tema es en el papel que juegan los factores «estresantes» laborales ${ }^{30,35}$. La mayoría de los profesionales encuestados manifestaban claramente la existencia de conflictos en relación con estos factores, que pueden ser los responsables mayoritarios de su insatisfacción ${ }^{5,23,32,33,36}$. Así, los profesionales que hallaban su labor poco reconocida tanto por los enfermos como por la dirección del centro presentaban las peores puntuaciones en el Burnout y en sus subescalas. A mayor abundamiento, existía una relación entre la opinión manifestada sobre la política de personal del centro y su propia identificación con el mismo, y puntuaciones significativamente más altas de agotamiento, despersonalización y Burnout, y más bajas de realización personal.

Si queremos buscar una relación entre este estado de insatisfacción y el síndrome de Burnout no podemos olvidar lo difícil que resulta encontrar datos científicos que expliquen la etiología del síndrome del «quemado». Así, es justo mencionar el trabajo de Wolpin y $\mathrm{col}^{37}$ en el que se demuestra que el Burnout causa bajos niveles de satisfacción 
laboral y no viceversa, siendo, según estos autores, por tanto la insatisfacción una consecuencia más del síndrome. Además, el fenómeno Burnout tiene mayores repercusiones y consecuencias negativas que la insatisfacción laboral, ya que incluye importantes cambios en la conducta hacia los beneficiarios de su trabajo, junto con una serie de manifestaciones sobre la calidad y desarrollo de las tareas que no se dan en la insatisfacción laboral.

Uno de los mayores problemas de la investigación actual en este tema es determinar cómo encaja el síndrome de Burnout dentro del paradigma del estrés ocupacional $^{6}$. Algunos autores ${ }^{1,4}$ apoyan la idea de que el Burnout es una respuesta al estrés crónico de los profesionales «de ayuda a los demás», de tal manera que datos aportados por ciertos investigadores ${ }^{38}$ afirman que el 90\% de las personas que experimentan Burnout manifiestan síntomas de excesivo estrés. Tal planteamiento ignora el gran número de variables que median entre estrés y Burnout, asumiendo que todo profesional estresado inevitablemente caería en Burnout, lo cual no es cierto al $100 \%$.

Otros autores que han abordado el tema de los factores estresantes laborales en los profesionales sanitarios ${ }^{33}$, concluyen que las características sociodemográficas y laborales de los sujetos más susceptibles al estrés son radicalmente diferentes del perfil asociado al síndrome de Burnout obtenido en nuestro estudio y en otros semejantes.

Trabajos que tratan específicamente del problema del estrés en enfermería hablan de un claro predominio de la sobrecarga de trabajo y la inexperiencia de los trabajadores como causas básicas de la aparición del mismo, mientras que los profesionales con mayor edad y antigüedad en el servicio perciben con menor frecuencia los factores estresantes laborales ${ }^{32,33}$.
Por el contrario nuestro estudio nos confirma cómo el perfil del sujeto afecto de Burnout se acerca a esta definición: profesional de enfermería con una antigüedad en el servicio de 15 años de media, y por lo tanto experimentado, y que manifiesta un escaso grado de reconocimiento de su labor, así como alto grado de insatisfacción con la gestión del centro de trabajo. Precisamente el bajo grado de reconocimiento de su labor profesional es destacado en el estudio realizado por Pérez Andrés y cols. como uno de los factores determinantes fundamentales del grado de satisfacción laboral de este colectivo $^{18}$.

Todo ello tiene más importancia, si cabe, si tenemos en cuenta que el síndrome de Burnout puede acarrear consecuencias graves, y no sólo estrés, al afectar al rendimiento y a la calidad de la labor profesional y a la salud física y mental del propio trabajador $4,15,23,39,40$.

Recientes publicaciones relacionan el síndrome del «quemado» con alteraciones somáticas graves, e incluso hablan de la aparición, en el Burnout crónico de altos niveles de cortisol en saliva durante la jornada de trabajo ${ }^{41-43}$. Estos cambios podrían explicar la asociación recientemente postulada del síndrome del «quemado» con la enfermedad cardiovascular ${ }^{44,45}$.

Por último, otros autores ${ }^{30,46}$ llegan a relacionar el Burnout y especialmente el componente de despersonalización con un déficit de la inmunidad celular, al detectar una disminución de las células NK (Natural killer) y baja proporción de CD57 y CD16 respecto al total de linfocitos, en los sujetos afectos por el proceso.

Por todo ello, queremos resaltar la importancia que el estudio en profundidad, del fenómeno Burnout y de sus factores desencadenantes, puede tener, ya que estos conocimientos redundarán en una mejora de la calidad de la asistencia sanitaria, con claras ven- 
tajas para los pacientes, los profesionales y por supuesto las instituciones implicadas ${ }^{47-50}$.

\section{AGRADECIMIENTOS}

Queremos expresar nuestro agradecimiento a D. Alfredo Moreno, director de enfermería, del Hospital Clínico Universitario San Carlos de Madrid, sin cuya colaboración este trabajo no hubiera podido realizarse.

\section{BIBLIOGRAFÍA}

1. Golembiewski RT, Munzenrider R. Phases of psychological burn-out and organizational covariant: a replication using norms from a large population. J Health Human Resourc Administrat 1984; 6: 290-323.

2. Leiter MP, Harvie P. Burnout among mental health workers: a review and a research agenda. Int J Soc Psychiatry $1996 ; 42: 90-101$

3. Edwards D, Burnard P, Coyle D, Fothergill A, Hannigan $\mathrm{B}$. Burnout in community mental health nursing: a review of the literature. J Psychiatr Ment Health Nurs 2000; 7:7-14

4. Maslach C, Jackson S. Maslach Burnout inventory. Palo Alto: Consulting Psychologist Press; 1986.

5. Bustinza A, López-Herce J, Carrillo A, Vigil MD, de Lucas N, Panadero E. Situación del Burnout de los pediatras intensivistas españoles. An Esp Pediatría 2000; 52: 418-23.

6. Oliver C. El Burnout como síndrome específico [tesis doctoral]. Madrid: Facultad de Psicología de la Universidad Complutense de Madrid; 1993.

7. Cherniss C. Staff Burnout: job stress in human services. Beverly CA: Sage; 1980.

8. Cherniss C. Beyond burnou: helping teachers, nurses, therapists, and law recover from form stress and disillusionment. New York: Routledge; 1985.

9. Nobel G, Lluch M.T, Miguel MD. Enfermería psicosocial 2. Barcelona: Salvat; 1991.

10. Demeuroti E, Bakker AR, Nachreiner F, Schaufeli WB. A model of Burnout and life satisfaction among nurses. J Adv Nurs 2000; 32: 454-64.
11. House JS. Work stress and social support. Addisson-Wesley: Reading Mas; 1980.

12. Atance JC. Aspectos epidemiológicos del síndrome de Burnout en personal sanitario. Rev Esp Salud Pública 1997; 71: 294-303

13. Lee V, Henderson MC. Occupational stress and organizational comitment in nurse administrators. J Nurs Administ 1996; 26:21-8.

14. Turnipseed DL . Ansiety and Burnout in the health care work environment. Psychol Report 1998; 82: 627-42.

15. Felton JS. Burnout as a clinical entity-its importance in health care workers. Occup Med (London) 1998; 48: 237-50

16. Constantini A, Solano L Di Napoli R, Bosco A. Relation between hardiness and risk of Burnout in a sample of 92 nurses working in oncology and AIDS wards. Psychotherapy \&Psychosom 1997; 66:78-82.

17. Freudenberg HJ . Staff burn-out. J Socl Issues 1974; 30: 159-65.

18. Pérez Andrés C, Alameda A, Albéniz C. La formación práctica en enfermería en la Escuela Universitaria de Enfermería de la Comunidad de Madrid. Opinión de los alumnos y de los profesionales asistenciales. Un estudio cualitativo con grupos de discusión. Rev Esp Salud Pública 2002;76:517-30.

19. Mira JJ, Vitaller J, Buil JA, Aranaz J, RodríguezMarín J. Satisfacción y estrés laboral en médicos generalistas del sistema Público de Salud. At Primaria 1994;14: 1135-40.

20. Orozco P, García E. Factores que influyen en el nivel de cansancio laboral de los médicos de atención primaria. Aten Primaria 1993;12:135-8.

21. De las Cuevas C, González de Rivera JL, De la Fuente JA, Alviani M, Ruiz-Benítez A. Burnout and reaction to stress. Rev Med Univ Navarra 1997;41:10-18.

22. Cebria J, Segura J, Corbella S, Sos P, Comas O, García M, Rodríguez C, Pardo MJ, Pérez J. Rasgos de personalidad y Burnout en médicos de familia. At Primaria 2001; 27:459-68.

23. Hidalgo I, Díaz RJ. Estudio de la influencia de los factores laborales y sociodemográficos en el desarrollo del síndrome de agotamiento profesional en el área de medicina especializada del Insalud de Ávila. Med Clin (Barc) 1994; 103:408-12. 
24. Moreno B, Oliver C, Pastor JC, Aragoneses A. El Burnout una forma específica de estrés laboral. En: Carballo VE \&Buela G, editores. Manual de psicología clínica. Madrid: Siglo XXI; 1991. p. 271-84.

25. Jackson SE, Schwab RL, Schuler RS. Toward on understanding of the Burnout phenomenon. J Appl Psychol 1986;4 :630-40.

26. De Pablo R, Suberviola JF. Prevalencia del síndrome de Burnout o desgaste profesional en los médicos de atención primaria. Aten primaria 1998; 22: 580-4.

27. Cano MA, García C, García E, López M, Parera N. ¿Está quemada la enfermería?: síndrome de burnout. Enf Intensiva 1996; 7:138-46.

28. Faura I, VendrellT. The Burnout síndrome. Nursing, a high-risk group. Rev Enf 1988; 11:53-6.

29. Kilfeldder CJ, Power KG, Wells TJ. Burnout in psychiatric nursing. J Adv Nurs 2001; 34:383-97.

30. Schnorpfeil P, Noll A, Wirtz P, Schulze R, Ehlert U, Frey K., Fischer JE. Assesment of exhaustion and related risk factors in employees in the manufacturing industry- a cross sectional study. Int Arch Occup Environ Health 2002; 75:535-40.

31. Freudenberg HJ, Richelson G. Burnout: the high cost of high achievement. Garden City NY: Anchor Press; 1980.

32. Hillhouse JJ, Adler CM. Investigating stress effect patterns in hospital staff nurses: results of a cluster analysis. Soc Sci Med 1997;45:1781-8.

33. Escribá V, Más R, Cárdenas M, Burguete D, Fernández R. Estresores laborales y bienestar psicológico. Impacto en la enfermería hospitalaria. Rev Rol Enf 2000; 23:506-11

34. Gómez P, Santano A, Matías S, Roca S, Tomey MJ. Study of the work climate in four critical care units in a hospital. Enf Intensiv 1999; 10:120-8.

35. Rafferty Y, Firend R, Landsbergis PA. The association between job skill discretion, decision, authority and Burnout. Work\&Stress 2001; 15:73-85.

36. Revicki DA, May HJ. Organizational characteristics, occupational stress and mental health in nurses. Behavior Medicin 1989;15: 30-6.

37. Wolpin J, Burke RJ, Greenglass ER. Golembieski's phase model of psychological Burnout: some issues. Psychol Report 1990;66:451-7.

38. Belcastro PA, Gold RS. Teacher stress and burnout implications for school health personnel. J School Health 1983; 53: 404-7
39. Hare J, Pratt CC, Andrews D. Predictors of Burnout in professional and paraprofessional nurses working in hospitals and nursing homes. Int $\mathrm{J}$ Nurs Stud 1988; 25: 105-15.

40. Gundersen L . Physician Burnout. Ann Intern Med 2001; 135:145-8.

41. Melamed S, Kushnir T, Shirom A. Burnout and risk factors for cardiovascular diseases. Behavior Med 1992; 18: 53-60.

42. Thommasen HV, Connelly I, Lavanchy M, Berkowitz J, Grzybowsky S. Short report: Burnout, depression, and moving away. How are they related? Can Famill Physician 2001;47: 747-9.

43. Patachioli FR, Angelucci L, Dellerba G, Monnazzi P, Leri O. Actual stress, psychopathology and salivary cortisol levels in the irritable bowel syndrome (IBS). J Endocrinol Investig 2001; 24:173-9.

44. Melamed S, Ugarten U, Shirom A, Kahana L, Lerman Y, Froom P. Chronic burnout, somatic arousal and elevated salivary cortisol levels. J Psychosom Res 1999;46:591-8.

45. Van Amelsvoort LGPM, Schouten EG, Maan AC, Sweene CA, Kook FJ. Occupational determinants of heart rate variability. Int Arch Ocup Env Health 2000; 73:255-62.

46. Nakamura H, Nagase H, Yoshida M, Ogino K Natural Killer (NK) cell activity and NK cell subsets in workers with a tendency of Burnout. J Psychosom Res 1999;46:569-78.

47. López-Castillo J, Gurpegui M, Ayuso-Mateos JL, Luna JD, Catalán J. Emotional distress and occupational Burnout in health care professionals serving HIV-infected patients: a comparison with oncology and internal medicine services. Psychotherapy \& Psychosom 1999; 68, 348-56.

48. Laschinger HK, Finegan J, Shamian J, Wilk P Impact of structural and psychological empowerment on job strain in nursing work settings: expanding Kanter's model. J Nurs Administ 2001; 31: 260-72.

49. Ewers P, Bradshaw T, McGowern J, Ewers B. Does training in psychosocial interventions reduce Burnout rates in forensic nurses?. J Adv Nurs 2002; 37: 470-6.

50. Meier DE, Back AL, Morrison RS. The inner life of physicians and care of the seriously ill. JAMA 20001; 286: 3007-14 